\title{
Focal Temporoparietal Slow Activity in Alzheimer's Disease Revealed by Magnetoencephalography
}

\author{
Alberto Fernández, Fernando Maestú, Carlos Amo, Pedro Gil, Thorsten Fehr, \\ Christian Wienbruch, Brigitte Rockstroh, Thomas Elbert, and Tomás Ortiz
}

\begin{abstract}
Background: Patients suffering from Alzheimer's disease exhibit more activity in the conventional electroencephalographic delta and theta bands. This activity concurs with atrophy and reduced metabolic and perfusion rates, particularly in temporoparietal structures.
\end{abstract}

Methods: Whole-head magnetoencephalographic recordings were obtained from 15 patients diagnosed with Alzheimer's disease and 19 healthy control subjects during a resting condition. The generators of focal magnetic slow waves were located employing a single moving dipole model.

Results: Dipole density in the delta and theta bands was enhanced in the Alzheimer's disease group compared with healthy control subjects. Slow-wave activity differed significantly between groups in temporoparietal regions of both hemispheres. Right temporoparietal slow-wave activity covaried with cognitive performance, whereas left temporal delta activity varied with a functional status scale.

Conclusions: Our results support the predominant role of the temporoparietal areas in the diagnosis of Alzheimer's disease. Magnetoencephalography and the source analysis of focal slow activity in particular provide interesting and potentially clinically useful tools to assess functional modifications of patients' brain and to evaluate its relationship with the cognitive status. Biol Psychiatry 2002; 52:764-770 @ 2002 Society of Biological Psychiatry

Key Words: Alzheimer's disease, magnetoencephalography, delta, theta, focal, dementia

\section{Introduction}

$\mathrm{A}$ lzheimer's disease (AD) is one of the most prominent neurodegenerative diseases. Despite its clinical relevance, well-defined diagnostic criteria, derived from neu-

From the Centro MEG Dr. Pérez Modrego, Universidad Complutense de Madrid (AF, FM, CA, TO), Madrid, Spain; Servicio de Geriatría, Hospital Clínico Universitario San Carlos de Madrid, Madrid, Spain (PG); University of Konstanz and Center for Psychiatry (TF, CW, BR, TE), Reichenau, Konstanz, Germany.

Address reprint requests to Professor Dr. Tomás Ortiz Alonso, Centro MEG Dr. Pérez-Modrego, Facultad de Medicina, Pabellón 8, Universidad Complutense de Madrid, 28040 Madrid, Spain.

Received October 24, 2001; revised January 30, 2002; accepted February 7, 2002 rophysiologic or neuroradiologic-neurometabolic methods are still insufficient. Thus, a strict diagnosis of early $\mathrm{AD}$ compared with other degenerative disorders based on electroencephalography (EEG), magnetic resonance imaging (MRI), single-photon emission computed tomography (SPECT), or positron emission tomography (PET) is uncertain (for a review, see Rosén 1997). Summarizing key features from morphologic-functional studies, Yamaguchi et al (1997) concluded that 1) patients with $\mathrm{AD}$ present with a higher level of general brain atrophy than healthy aged subjects; 2) the hippocampus and the temporal lobe are usually the most atrophied areas of the brain in AD patients (although some authors, such as Thompson et al (2001) have highlighted the critical role of the parietal atrophy); 3) the cerebral blood flow (CBF) and the metabolic rate for oxygen $\left(\mathrm{CMRO}_{2}\right)$ are lower in patients with $\mathrm{AD}$ than in control subjects, especially in the temporo-parioto-occipital area; and 4) the cerebral metabolic rate for glucose (CMRGlc) is lower in the temporoparietal area in patients with $\mathrm{AD}$ compared with healthy aged control subjects.

The EEG literature indicates that a fifth feature might be added to this list: an increase of the absolute delta and theta power in the $\mathrm{AD}$ patients, usually focused in the temporoparietal regions (Buchan et al 1997; Dierks et al 2000). Both EEG and metabolic measures reflect, in essence, synaptic activity. The most common quantification procedure for EEG is spectral analysis by fast Fourier transformation (FFT) which depends on a "recording reference" point. Different references, however, will lead to different results. Furthermore, because of the distortion of volume currents by body tissue with varying conductivities, EEG-based source localization is cumbersome and its precision quite limited. These problems can be overcome by magnetic source imaging (MSI), which combines high temporal and high spatial resolution and allows detection of focal neural activity at intervals where simultaneous background activity is low. Using simple electromagnetic models, such as that from a single focal generator in one brain region, we can test how well the data fit this model; with a good fit, the generator can be localized with high precision and the localization superimposed 
onto MR slices (Elbert 1998). This technique can be used to localize evoked and also spontaneous magnetic activity (for a review, see Lewine and Orrison 1995a).

Few studies have employed MSI to investigate AD (Berendse et al 2000; Pekkonen et al 1996, 1999; Ribary et al 1991). Berendse et al (2000) published one of the first papers using magnetoencephalography (MEG) to measure spontaneous brain activity in AD patients. The authors concluded that absolute power in certain bands and the inter- and intrahemispheric coherence may assist in distinguishing $\mathrm{AD}$ from healthy control subjects. Their results, are consistent with EEG data and demonstrate, with few exceptions, lower coherence values in the AD group and an increase in low frequency power values that are restricted to frontal and central areas in the $4 \mathrm{~Hz}$ band.

Following previous studies on the clinical application (i.e., brain tumors, CVA, epilepsy) of frequency-band and Dipole Density analysis (de Munck et al 2001; de Jongh et al 2001; Lewine and Orrison 1995b; Vieth et al 1995, 2000;); Fehr et al (2001) advanced a methodology that allows the source localization of focal slow waves. Determining the focal dipole density of delta and theta activity, they observed a higher frequency of slow-wave generators, particularly in temporal regions, of schizophrenic patients compared with control groups. These authors considered slow activity as an indication of dysfunctional areas characterized by the clustering of slow-wave generators in the delta band.

Given this background, our study examined focal slowwave activity in $\mathrm{AD}$ patients. We hypothesized that focal slow-wave generators are abnormally frequent in the cortical structures, particularly in the parietal and temporal lobes, of patients with an AD diagnosis. Such an enhanced density of focal slow-wave generators could be the functional counterpart of the neurodegenative process associated with the disease.

\section{Methods and Materials}

\section{Subjects}

Fifteen patients (nine women) from the Hospital Universitario San Carlos de Madrid Geriatry unit fulfilling the NINCDSADRDA (National Institute of Neurological and Communicative Disorders and Alzheimer's Disease and Related Disorders Association) (McKhann et al 1984) criteria of probable AD and 19 control subjects (11 women) participated in this study after signing an informed consent. In cases of legal incapacity, a legal representative signed the consents. All subjects had general medical, neurologic, psychiatric, and psychologic examinations and participated in the neuroimaging studies. Prior to the MEG recording, all subjects signed an informed consent that explained the technical and ethical considerations of the technique. The study was approved by the ethical committee of the university.

All subjects underwent three additional tests: Functional Assessment Staging Test FAST was endorsed by several geriatri-
Table 1. Means and Standard Deviations for Age, CAMCOG, MMSE, and FAST Variables for Alzheimer's Disease (AD) and Control (C) Groups

\begin{tabular}{lll}
\hline & Mean & SD \\
\hline AGE & & \\
AD & 76.27 & 1.21 \\
C & 73.68 & 1.32 \\
CAMCOG & & \\
AD & 55.66 & 6.01 \\
C & 80.89 & 7.83 \\
MMSE & & \\
AD & 19.93 & 2.28 \\
C & 31.36 & 2.49 \\
FAST & & .5 \\
AD & 3.46 & .59 \\
C & 1.36 & \\
\hline
\end{tabular}

CAMCOG, cognitive portion of Cambridge Examination for Mental Disorders of the Elderly; MMSE, Mini-Mental State Examination (Spanish version); FAST, Functional Assessment Staging Test.

cians (FAST; Reisberg 1998) to evaluate the functional states, the CAMCOG (cognitive) part of the Cambridge Examination for Mental Disorders of the Elderly (CAMDEX; Roth et al 1988) and the Spanish version of the Mini-Mental State Examination (MMSE; Lobo et al 1979) to assess cognitive functions. Table 1. summarizes subjects' demographic and cognitive/functional status.

\section{Data Collection}

The MEG was measured using a 148-channel whole-head magnetometer (MAGNES 2500 WH,4D Neuroimaging., San Diego, CA) during a 10-min resting period. While lying comfortably on a patient bed, subjects were asked to stay awake and to avoid head and eye movement by fixating on a black point on the ceiling. The MEG was recorded with a $678.17-\mathrm{Hz}$ sampling rate, using a band-pass filter of 0.1 to $200 \mathrm{~Hz}$. Eye movements (EOG) were recorded from four electrodes attached to the left and right outer canthus and above and below the left eye. The electrocardiogram (ECG) was monitored with electrodes attached to the right collarbone and the lowest left rib.

\section{Data Reduction and Analysis}

Each 10-min data set was decimated (which consisted of filtering the data to respect Nyquist criteria [Oppenhein and Schafer 1974], followed by a downsampling by factor of 16) and band-pass filtered before the analysis in the delta $(1.5-4.0 \mathrm{~Hz})$ and theta $(4.0-8.0 \mathrm{~Hz})$ bands. Dipole densities were estimated based on an equivalent current dipole in a homogeneous sphere calculated for each time step. The data and dipole density analysis followed Fehr et al (2001).

\section{Dipole Density Analysis}

Artifact-free time segments of varying lengths were determined by visual inspection. Single equivalent dipoles were fitted for each time point in the selected epochs. Only dipole fit solutions at time points with a root mean square $\left[\mathrm{RMS}=\left(\times 1 / \mathrm{n} \times\left(\mathrm{x}_{\mathrm{i}}\right)^{2}\right)\right)$ ] 

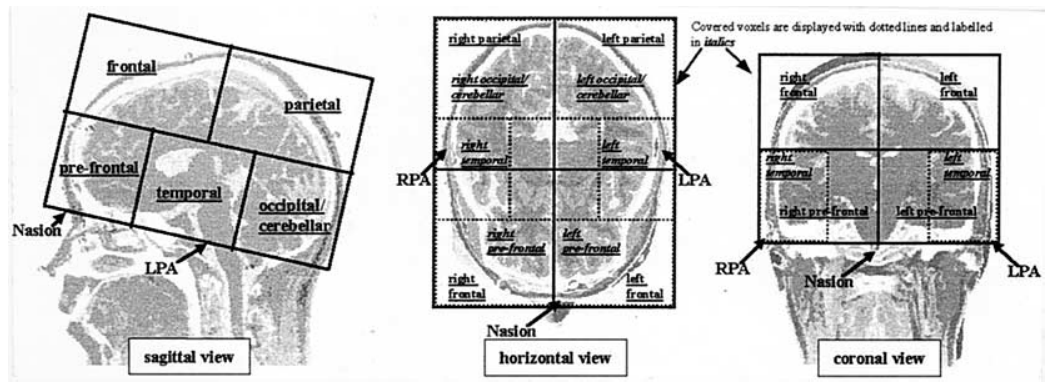

Figure 1. Schematic overview of the regions used to calculate dipole density solutions in statistics. over $100 \mathrm{fT}$ and a goodness of fit (GOF) over 0.90 were accepted for further analysis. For statistical analysis, the total brain volume was divided into 10 regions, representing frontal, parietal, prefrontal, temporal, and occipital areas in both hemispheres, and the number of successful dipole fits was determined for each of these regions. The total number of dipoles per second fitting the criteria in the delta and theta bands was determined for each subject and region. To avoid the possible influence of the individual time-segment lengths over the dipole density scores, the raw number of dipoles per region were normalized by calculating the formula: number of dipoles per second $=$ raw number of dipoles/sampling rate $\times$ absolute number of recorded points (where the absolute number of recorded points is the final number of recorded points after eliminating the artifacts). This "number of dipoles per second per region" is the final score used for the statistical analysis (Figure 1).

Differences of the dipole density solutions between groups (AD and control) were evaluated by means of a $2 \times 10$ (group $\times$ region) repeated measures analysis of variance (ANOVA). In cases of statistical significance, the pairwise mean comparison was carried out by Tuckey-b post hoc analysis.

The relationship between the cognitive (CAMCOG, MMSE) and functional (FAST) evaluations and the variations of neuromagnetic activity were also evaluated. First, a correlation analysis was made over the three cognitive-functional and the 10 neuromagnetic-regional variables. Then, multiple linear regression was carried out to define the predictability of the relationship between the 10 neuromagnetic-regional variables and the

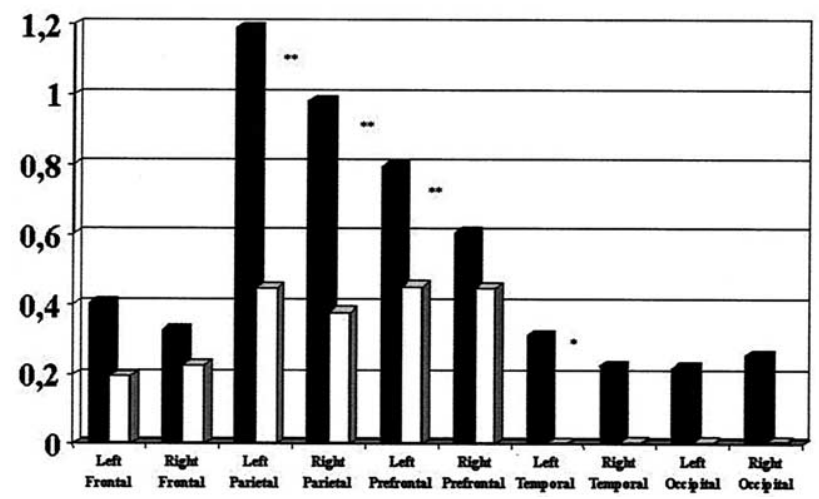

Figure 2. Region $\times$ group effect in delta band. The y axis shows the number of dipoles per second in every region. Black bars, AD group; white bars, control group. Significant differences are indicated by asterisks: $* p<.05 ; * * p<.01$. three cognitive-functional ones. All of these data analyses were made using the SPSS 8.0 statistical package.

\section{Results}

\section{Analyses of Variance for Dipole Density Data}

Figures 2 and 3 display the number of dipoles fitted per second in each region for delta and theta bands. For delta band, the density of focal slow waves demonstrates a significant main effect of group factor $[F(1,32)=6.47$; $p<.05$ ], with an increase of slow activity in the AD group in specific regions. This effect was confirmed by the significant interaction group $\times$ region $[F(9,288)=2.43$; $p<.05]$. In this case, the results demonstrate that the increase of delta activity in the AD group was higher over four specific regions: left parietal $(q=7.57 ; p<.01)$, right parietal $(q=6.15 ; p<.01)$, left temporal $(q=2.6$; $p<.05)$, and left prefrontal $(q=3.46 ; p<.01)$. Finally, a significant main effect of region factor, independent of the group factor, also appeared $[F(9,288)=13.021 ; p<$ $.001]$, showing a higher level of slow activity over prefrontal and parietal regions.

A similar pattern appears in the theta band. The significant main effect of group $[F(1,32)=8.17 ; p<.01]$ and

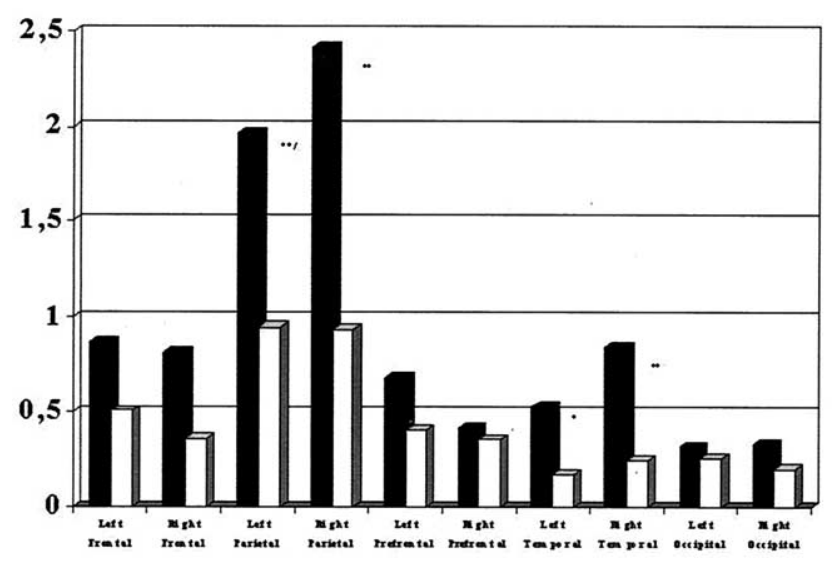

Figure 3 . Region $\times$ group effect in theta band. The y axis shows the number of dipoles per second in every region. Black bars, AD group; white bars, control group. Significant differences are indicated by asterisks: $* p<.05 ; * * p<.01$. 


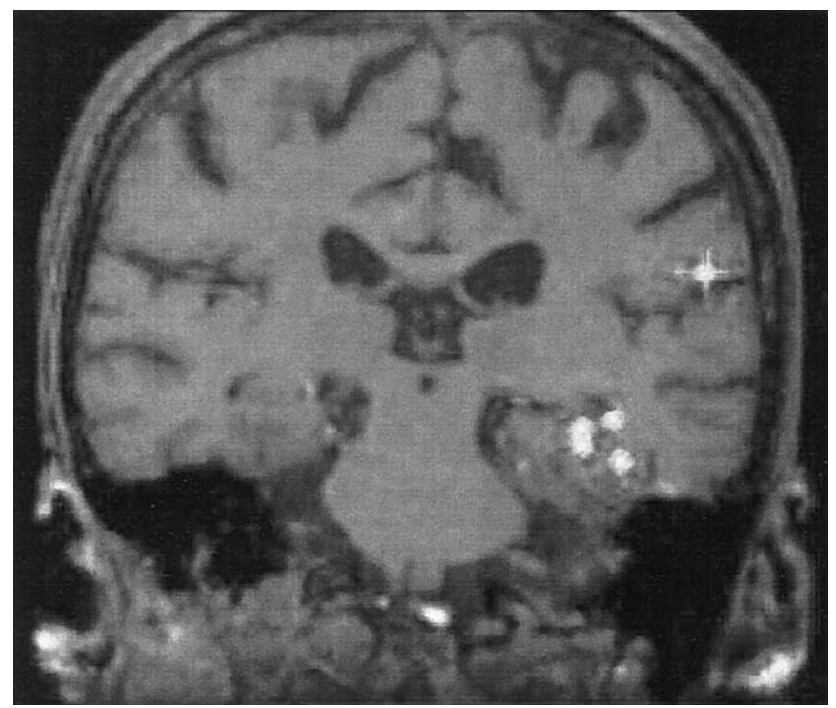

Figure 4. Predominant left temporoparietal slow-wave activity in an Alzheimer's disease patient, represented by white full circles.

the interaction of this variable with region $[F(9288)=$ 3.02; $p<.01]$ demonstrates again a general slowing in the AD group, more evident in the left parietal $(q=5.6 ; p<$ $.01)$, right parietal $(q=8.24 ; p<.01)$, left temporal $(q=$ $2.02 ; p<.05)$, and right temporal $(q=3.25 ; p<.01)$ regions. In this case, the region main effect $[F(9,288)=$ $14.81 ; p<.001]$ indicates a strong increase in theta activity in parietal areas, independently of the group. Figure 4. shows an example of left-predominant temporoparietal slow-wave activity in an AD patient.

\section{Correlation and Multiple Linear Regression Analysis}

Pearson's correlation coefficients were calculated for the 10 anatomic regions, both in delta and theta bands, and the three cognitive-functional variables. The MMSE scores varied with higher left parietal $(r=-3.5 ; p<.05)$, right parietal $(r=-0.37 ; p<.05)$, and left temporal $(r=$ $-0.34 ; p<.05)$ delta dipole density and with right parietal $(r=-0.35 ; p<.05)$ and right temporal $(r=$ $-0.38 ; p<.05)$ theta activity. The CAMCOG scores correlated with right parietal $(r=-0.43 ; p<.01)$ and left temporal $(r=-0.38 ; p<.05)$ delta activity; and with right parietal $(r=-0.36 ; p<.05)$ and right temporal $(r=$ $-0.37 ; p<.05)$ theta activity. Finally, FAST correlated only with left temporal delta activity $(r=0.36 ; p<.05)$ and right parietal theta activity $(r=0.34 ; p<.05)$.

The multiple linear regression analysis indicates that only right parietal delta activity predicts the variability of CAMCOG scores $[F(1,37)=8,32 ; p<.01]$, with higher right parietal activity predicting a lower CAMCOG score $(t=-2.9 ; p<.01)$ and therefore worse patient mental status. A significant regression effect was found for the MMSE score $[F(1,37)=6.3 ; p<.05]$, illustrating that right temporal theta activity predicts the variability of MMSE scores $(t=-2.5 ; p<.01)$, with more pronounced theta activity predicting a lower MMSE score and thus worse patient mental status. For FAST, the regression effect was also significant $[F(1,37)=5.531 ; p<.05]$. Left temporal delta activity predicted FAST score variability $(t=2.23 ; p<.05)$; the higher the left temporal slow activity, the higher the FAST score and thus the lower the patient's functional status.

\section{Discussion}

The results of our study essentially confirm our hypothesis: AD patients exhibited more MEG delta and theta activity than healthy aged control subjects, because the focal slow waves would be focused on temporal and parietal structures. Our data are consistent with previous results from EEG analyses (Claus et al 1998, 2000; Erkinjuntti et al 1988; Huang et al 2000; Jelic et al 2000; Wada et al 1997). It should be noted that EEG does not allow comparable source localization and that tangentially oriented dipoles may produce distant scalp activity; for example, when located in temporal areas, activity in the EEG amplitude may become largest over frontal scalp regions because of the nature of the volume conduction.

Several authors (Dringenberg 2000; Lehtovirta et al 2000; Riekkinen et al 1991) have explained the slowing of $\mathrm{AD}$ magnetoelectric activity by the so-called cholinergic hypothesis. Longo (1966) showed that systemic injections of cholinolytics induce slow delta activity in humans and animals. Shute and Lewis (1967) confirmed this pharmacologic evidence, finding an association between the cholinergic-dependent ascending reticular system and neocortical EEG activity (Riekkinen et al 1991). The reduction of cholinergic levels (in terms of decreased AChE in the cerebrospinal fluid [CSF]) and the atrophy of the cholinergic nucleus basalis of Meynert (NBM), the major source of ascending cholinergic afferents (Riekkinen et al 1991), are characteristics of AD patients that correlate with the severity of dementia (Arendt et al 1984). Reiekkinen et al (1990) detected a significant correlation between AChE activity of the CSF and the delta power in patients with $\mathrm{AD}$ but did not find any correlation between metabolic markers for dopamine, noradrenalin, or serotonin. They also observed in autopsy studies that patients with the highest EEG delta power had an extremely low cell density in the NBM and low AChE activity in the cortex. In sum, the most prominent symptoms of $\mathrm{AD}$ (i.e., loss of memory and spatial abilities) appear to be closely related to the slowing of the magnetoelectric activity and 
to a reduction of cholinergic brain levels (Penttilä et al 1985).

Our results are also consistent with regional PETSPECT or MRI findings. In a comprehensive review, Rapoport (1991) pointed out that regional percentage reductions in cerebral metabolism consistently identified the parietal and temporal cortices as being most severely affected in patients with $\mathrm{AD}$, whereas the frontal or occipital cortices were less involved. This line of evidence was confirmed in further studies (Jelic and Nordberg 2000; Muller et al 1999; Nagata et al 2000; Small et al 2000). These studies revealed the usefulness of temporoparietal metabolic scores for making a differential diagnosis of $\mathrm{AD}$ and the association of this abnormal pattern of regional metabolism with genetic risk factors. Congruent results are obtained by MRI research. The traditional approach focused on hippocampal, parahippocampal, and temporolateral atrophy (Barber et al 2001; Foundas et al 1997; Galton et al 2001). Recently, Thompson et al (2000), using detailed maps of cortical gray matter loss in $\mathrm{AD}$, demonstrated the critical contribution of bilateral, but predominantly left-sided, temporoparietal atrophy. In this study, the temporal and temporoparietal cortices exhibited severe (up to $30 \%$ ) reduction in gray matter in the AD patients. This loss contrasted with a comparatively intact central and precentral gyri and the occipital lobes $(0-5 \%)$, for example.

This metabolic, structural, and magnetoelectric evidence highlights the role of temporoparietal cortex in the diagnosis of AD. The potential and more accurate role of these techniques depends on their ability not only to classify $\mathrm{AD}$ subjects and control subjects but also to predict the cognitive and functional status and the survival rate of these patients. Some early attempts (Naguib and Levy 1982) using computerized tomographic scans showed that the parietal rate of radiologic density was an indication of poor prognosis in AD patients. More recent MRI data (Killiany et al 2000) confirmed that structural measurements of the entorhinal cortex and the banks of the superior temporal sulcus predict the development of $\mathrm{AD}$ in a group of 119 elderly individuals. Two SPECT studies revealed very similar findings (Claus et al 1999; Jagust et al 1998). Jagust's group used regional cerebral blood flow $(\mathrm{rCBF})$ as predictors of survival in a life-table analysis for $\mathrm{AD}$ patients. Only right parietal $\mathrm{rCBF}$ was a significant predictor of survival, with subjects in the lowest tertile having shortest survivals. Claus et al (1999) demonstrated that lower left temporoparietal $\mathrm{rCBF}$ was significantly related to decline in language function. Left temporal rCBF was also considered as a threshold factor to predict the survival of the AD group. The lowest quartile scores predicted the survival time, with a mean of 2.7 years. Finally, Nobili et al (1999) investigated whether conven- tional EEG-bands assessed in a quantitative way could be used to estimate the time of arrival of patients with $\mathrm{AD}$ at three functional end points (loss of activities of daily living, incontinence, and death). They found that bilateral delta relative power yielded the maximum likelihood for estimating the onset of loss of activities of daily living in the $\mathrm{AD}$ group, right-side delta relative power was significantly related to onset of incontinence, and left-side delta relative power predicted the timing of death.

Our results confirm that temporoparietal dysfunction can predict the cognitive and functional status of patients with AD. Right parietal delta activity was significantly associated with CAMCOG scores, whereas left temporal theta activity predicted the variability of MMSE results. Both tests assess functional status; therefore, the increase of slow activity predicts the mental capability of these patients. More important from our point of view is the confirmed association of left temporal delta activity with FAST scores. The FAST (Sclan and Reisberg 1992) is a seven-stage scale designed, and strongly endorsed, for testing AD patients. FAST stages offer a portrait of the cognitive level of the patient and an estimation of the ongoing functional deterioration. Patients at stages 3 and 4 have been shown to manifest clear deterioration characteristics of dementia over subsequent intervals of 2 years. Thus, higher left temporal delta magnetic activity can predict the functional situation of the patient and the risk of developing dementia.

Further longitudinal studies should be carried out to establish the predictive diagnostic power of slow-wave magnetic activity in AD patients. Our results suggest a potential diagnostic and prognostic value of the dipoledensity analysis.

This research was supported by $4 \mathrm{D}$-Neuroimaging and the Deutsche Forschung Gemeinschaft (DFG).

\section{References}

Arendt T, Bigl V, Walther F, Sonntag M (1984): Decreased ratio of CSF acetylcholinesterase to butylcholinesterase activity in Alzheimer's disease. Lancet 1:173.

Barber R, McKeith IG, Ballard C, Gholkar A, O'Brien JT (2001): A comparison of medial and lateral temporal lobe atrophy in dementia with Lewy bodies and Alzheimer's disease: Magnetic resonance imaging volumetric study. Dement Geriatr Cogn Disord 12:198-205.

Berendse HW, Verbunt JPA, Scheltens P, Van Dijk BW, Jonkman EJ (2000): Magnetoencephalographic analysis of cortical activity in Alzheimer's disease: A pilot study. Clin Neurophisiol 111:604-612.

Buchan RJ, Nagata K, Yokoyama E, Langman H, Yuya H, Hirata Y, et al (1997): Regional correlations between the EEG and 
oxygen metabolism in dementia of the Alzheimer's type Electroencephalogr Clin Neurophysiol 103:409-417.

Claus JJ, Kwa VI, Teunisse S, Walstra GJ, Van Gool WA, Koelman JH, et al (1998): Slowing on quantitative spectral EEG is a marker for rate of subsequent cognitive and functional decline in early Alzheimer disease. Alzheimer Dis Assoc Disor 12:167-174.

Claus JJ, Ongerboer De Visser BW, Bour LJ, Walstra GJ, Hijdra A, et al (2000): Determinants of quantitative spectral electroencephalography in early Alzheimer's disease: Cognitive function, regional cerebral blood flow, and computed tomography. Dement Geriatr Cogn Disord 11:81-89.

Claus JJ, Walstra GJM, Hijdra A, Van Royen EA, Verbeeten Jr B, Van Gool WA (1999): Measurement of temporal regional cerebral perfusion with single photon emission tomography predicts rate of decline in language function and survival in early Alzheimer's disease. Eur J Nucl Med 26:265-271.

de Jongh A, de Munck JC, Baayen JC, Jonkman EJ, Heethaar RM, Van Dijk BW (2001): The localization of spontaneous brain activity: First results in patients with cerebral tumors Clin Neurophysiol 112:378-385.

de Munck JC, de Jongh A, Van Dijk BW (2001): The localization of spontaneous brain activity: An efficient way to analyze large data sets. IEEE Trans Biomed Eng.

Dierks T, Jelic V, Pascual-Marqui RD, Wahlund LO, Julin P, Linden DEJ, et al (2000): Spatial pattern of cerebral glucose metabolism (PET) correlates with localization of intracrebral EEG-generators in Alzheimer disease. Clin Neurophysiol 111:1817-1824.

Dringenberg HC (2000): Alzheimer's disease: More than a "cholinergic disorder"-evidence that cholinergic-monoaminergic interactions contribute to EEG slowing and dementia. Behav Brain Res 115: 235-249.

Elbert T (1998): Neuromagnetism. In: Andrä W, Nowak H, editors. Magnetism in Medicine. New York. John Wiley and Sons, pp 190-262.

Erkinjuntii T, Larsen T, Sulkava R, Ketonen L, Laaksonen R, Palo J (1988): EEG in the differential diagnosis between Alzheimer's disease and vascular dementia. Acta Neurol Scand 77:36-43.

Fehr T, Kissler J, Moratti S, Wienbruch C, Rockstroh B, Elbert $\mathrm{T}$ (in press): Source distribution of neuromagnetic slow waves and MEG-delta activity in schizophrenic patients. Biol Psychiatry.

Foundas AL, Leonard CM, Mahoney SM, Agee OF, Heilman KM (1997): Atrophy of the hippocampus, parietal cortex, and insula in Alzheimer's disease: A volumetric magnetic resonance imaging study. Neuropsychiatry Neuropsychol Behav Neurol 10:81-89.

Galton CJ, Gomez-Anson B, Antoun N, Scheltens P, Patterson $\mathrm{K}$, Graves M, et al (2001): Temporal lobe rating scale: Application to Alzheimer's disease and frontotemporal dementia. J Neurol Neurosurg Psychiatry 70:165-173.

Huang C, Wahlund L, Dierks T, Julin P, Winblad B, Jelic V (2000): Discrimination of Alzheimer's disease and mild cognitive impairment by equivalent EEG sources: A crosssectional and longitudinal study. Clin Neurophysiol 111: 1961-1967.

Jagust WJ, Haan MN, Reed BR, Eberling JL (1998): Brain perfusion imaging predicts survival in Alzheimer's disease. Neurology 51:1009-1013.
Jelic V, Johansson SE, Almkvist O, Shigeta M, Julin P, Nordberg A, et al (2000): Quantitative electroencephalography in mild cognitive impairment: Longitudinal changes and possible prediction of Alzheimer's disease. Neurobiol Aging 21:533540.

Jelic V, Nordberg A (2000): Early diagnosis of Alzheimer disease with positron emission tomography. Alzheimer Dis Assoc Disord 14(suppl 1):S109-S113.

Killiany RJ, Gomez-Isla T, Moss M, Kikinis R, Sandor T, Jolesz F (2000): Use of structural magnetic resonance imaging to predict who will get Alzheimer's disease. Ann Neurol 47: $430-439$.

Lehtovirta M, Partanen J, Kononen M, Hiltunen J, Helisalmi S, Hartikainen P (2000): A longitudinal quantitative EEG study of Alzheimer's disease: Relation to apolipoprotein E polymorphism. Dement Geriatr Cogn Disord 11:29-35.

Lewine JD, Orrison WW (1995a): Magnetoencephalography and magnetic source imaging. In: Orrison WW, Lewine JD, Sanders JA, Hartshorne MF, editors. Functional Brain Imaging. St. Louis, MO: Mosby-Year Book, pp 404.

Lewine JD, Orrison WW (1995b): Spike and slow wave localization by magnetoencephalography. Neuroimaging Clin North Am 5:575-596.

Lobo A, Ezquerra V, Gomez Burgada F, Sala JM, Seva Diaz A (1979): El mini-examen cognoscitivo: Un test sencillo y práctico para detectar alteraciones intelectivas en pacientes médicos. Actas Luso Esp Neurol Psiquiatr Cienc Afines 3:189-202.

Longo VG (1966): Effects of scopolamine and atropine on electroencephalographic and behavioral reactions due to hypothalamic stimulation. J Pharmacol 116:198-208.

McKhann G, Drachman D, Folstein M, Katzman R, Price D, Stadlan EM, et al (1984): Clinical diagnosis of Alzheimer's disease: Report of NINCDS-ADRDA work group under the auspices of department of health and human services task force on Alzheimer's disease. Neurology 34:939-944.

Muller H, Moller HJ, Stippel A, Fric M, Grunwald F, Laux G, et al (1999): SPECT patterns in probable Alzheimer's disease. Eur Arch Psychiatry Clin Neurosci 249:190-196.

Nagata K, Maruya H, Yuya H, Terashi H, Mito Y, Kato H, et al (2000): Can PET data differentiate Alzheimer's disease from vascular dementia? Ann N Y Acad Sci 903:252-261.

Naguib M, Levy R (1982): Prediction of outcome in senile dementia: A computed tomography study. $B r J$ Psychiatry 140:263-267.

Nobili F, Copello F, Vitali P, Prastaro T, Carozzo S, Perego G, et al (1999): Timing of disease progression by quantitative EEG in Alzheimer's patients. J Clin Neurophysiol 16:566573.

Oppenhein AV, Schafer RW (1974): Digital Signal Processing. Englewood Cliffs, NJ: Prentice-Hall.

Pekkonen E, Houtilainen M, Virtanen J, Naatanen R, Ilmoniemi RJ, Erkinjuntii T (1996): Alzheimer's disease affects parallel processing between the auditory cortices. Neuroreport 31: $1365-1368$.

Pekkonen E, Jaaskelainen IP, Hietanen M, Huotilainen M, Naatanen R, Ilmoniemi RJ (1999): Impaired preconscious auditory processing and cognitive functions in Alzheimer's disease. Clin Neurophysiol 110:1942-1947. 
Pentilä M, Partanen VJ, Soininen H, Riekkinen PJ (1985): Quantitative analysis of occipital EEG in different stages of Alzheimer's disease. Electroencephalogr Clin Neurophysiol 60:1-6.

Rapoport SI (1991): Positron emission tomography in Alzheimer's disease in relation to disease pathogenesis: A critical review. Cerebrovasc Brain Metab Rev 3:297-335.

Reisberg B (1988): Functional assessment staging (FAST). Psychopharmacol Bull 24:653-659.

Ribary U, Ioannides AA, Singh KD, Hasson R, Bolton JP, Lado F, et al (1991): Magnetic field tomography of coherent thalamocortical 40-Hz oscillations in humans. Proc Nat Acad Sci USA 88:11037-11041.

Riekkinen P, Buzsaki G, Riekkinen P, Soininen H, Partanen J (1991): The cholinergic system and EEG slow waves. Electroencephalogr Clin Neurophysiol 78:89-96.

Riekkinen P Jr, Sirviö J, Riekinen PJ (1990): Relationship between EEG delta power and cortical ChAT content. Neurosci Res 8:12-20.

Rosén I (1997): Electroencephalography as a diagnostic tool in Dementia. Dement Geriatr Cogn Disord 8:110-116.

Roth M, Huppert FA, Tym E, Mountjoy CQ (1988): CAMDEX, the Cambridge Examination for Mental Disorders of the Elderly. Cambridge, UK: Cambridge University Press.

Sclan SG, Reisberg B (1992): Functional assessment staging (FAST) in Alzheimer's disease: Reliability, validity, and ordinality. Int Psychogeriatr 4:55-69.

Shute CCD, Lewis PR (1967): The ascending cholinergic reticular systems: Neocortical, olfactory and subcortical projections. Brain 90:497-520.
Small GW, Ercoli LM, Silverman DH, Huang SC, Komo S, Bookheimer SY, et al (2000): Cerebral metabolic and cognitive decline in persons at genetic risk for Alzheimer's disease. Proc Nat Acad Sci USA 97:6037-6042.

Thompson PM, Mega MS, Woods RP, Zoumalan CI, Lindshield CJ, Blanton RE, et al (2001): Cortical change in Alzheimer's disease detected with a disease-specific population-based brain atlas. Cereb Cortex 11:1-16.

Vieth J, Kober H, Ganslandt O, Kamada K (2000): The clinical use of MEG activity associated with brain lessions. Presented at the 12th International Conference on Biomagnetism. Helsinki

Vieth J, Kober H, Grummich P (1995): Slow wave and beta wave activity associated with white matter structural brain lesions, localized by the dipole density plot. In: Baumgartner C, Deecke L, Stroink G, Willianson SJ, editors. Biomagnetism: Fundamental Research and Clinical Applications. Amsterdam: Elsevier/IOS-Press, 50-54.

Wada Y, Nanbu Y, Jiang ZY, Koshino Y, Yamaguchi N, Hashimoto T (1997): Electroencephalographic abnormalities in patients with presenile dementia of the Alzheimer type: Quantitative analysis at rest and during photic stimulation. Biol Psychiatry 15:217-225.

Yamaguchi S, Meguro K, Itoh M, Hayasaka C, Shimada M, Yamazaki H, et al (1997): Decreased cortical glucose metabolism correlates with hippocampal atrophy in Alzheimer's disease as shown by MRI and PET. J Neurol Neurosurg Psychiatry 62:596-600. 